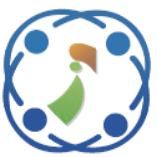

\title{
Uncertain Multi Level Four Dimensional Multi Item Fractional Transportation Problem
}

\author{
A. N. Revathi ${ }^{1}$ \\ S. Mohanaselvi ${ }^{1 *}$ \\ ${ }^{1}$ Department of Mathematics, Faculty of Engineering and Technology, \\ SRM Institute of Science and Technology, Kattankulathur, Chennai-603 203, Tamil Nadu, India. \\ * Corresponding author's Email: mohanass@srmist.edu.in
}

\begin{abstract}
This work will be illustrating a model for dealing with multi level four dimensional fractional multi item transportation problem whose parameters in both objectives and constraints are considered as uncertain variables. The Fractional programming technique is useful in dealing with real life situations where the decision maker has to maximize or minimize the ratios of two objective functions. In any hierarchical system or decentralized system, multi level programming problem can be used to obtain efficient solution based on the preferences of each decision maker at each level. The usage of fractional method and level wise preference in real life problems helps us to obtain the efficiency of system and also enables hierarchical decision making which is common in day to day transportation firms. An equivalent deterministic model has been obtained for the multi level four dimensional fractional multi item transportation problem under uncertain environment by using expected and chance constrain model of uncertainty theory. Then the deterministic model is solved by using the modified fuzzy goal programming technique to obtain the compromise solution of the proposed model. The validity of the proposed model has been explained using the numerical example.
\end{abstract}

Keywords: Uncertain variable, Multi level transportation, Fractional programming, Fuzzy goal programming, Compromise optimal solution.

\section{Introduction}

Transportation problems play a crucial role in transportation planning for organisations to enhance profit and time constraint. In the growing global competition, it is very much important for every company to find a way for profitable operations by a well-defined mechanism. Also, during a transportation process, transporting two or more items simultaneously could prove more beneficial. Considering availability of a variety of different transportation options, could convert a transportation problem (TP) into a solid transportation problem (STP). Similarly, considering various routes available for reaching a destination, could open up more possibilities and opportunities for a profitable operation for the organisation. The above quest arises the need for considering more parameters in the objective functions apart from the traditional ones like different routes and transportation of multiple items simultaneously.

Therefore, accounting for multiple factors like origins, destinations, conveyances, multiple routes and multiple products converts a transportation problem into a four dimensional transportation problem (4DTP).

A fractional transportation problem (FTP) is a special case of TP consisting of an objective function in the term of ratio of two non-identical functions. The advantage of fractional programming is that the ratio of economical terms represent the efficiency, making it helpful for the decision maker to weigh choices.

In real life situations, the parameters such as transportation cost, time, demand and supply keep varying constantly due to many uncontrollable and non-human factors, leading to the study of transportation problems involving uncertainty. Liu, in 2015 , illustrated that estimating the probability distribution for parameters can prove very tough in the absence of historical information. Also, in many 
cases where fuzzy set theory was employed, counterintutive results were obtained. Therefore, an alternative way is seeking the help of experts for evaluation of an occurrence's belief degree; the belief degrees can be handled and manipulated using the uncertainty theory proposed by Liu in 2009, which was then improvised in 2010.

In this work, four dimensional multi item fractional transportation problem involving uncertain parameters has been formulated and solved considering a multi level heirarchical decision making system. In multi level programming problems the initial level decision maker solves the problem and passes the information on to the second level decision maker (DM) on the hierarchy. The second level DM uses the information passed on to him and solves the problem. Similarly, the DM at each level tries to maximize or minimize the objective function, using the variables which are generally impacted and constrained by the previous decision makers. The objective function's heirarchy represents the order of the preference of the decision makers concerning the respective objectives. Till date, the multi level four dimensional multi item fractional transportation problem under uncertain environment has not been formulated and investigated yet, as per the knowledge of the authors. The uncertain multi level four dimensional multi item fractional transportation problem (UML4DMIFTP) proposed in this paper is very much nearby the operational procedure of real life transportation firms. The aim of this paper is to present a method to solve uncertain ML4DMIFTP. Utilising expected value and chance constraint models on uncertainty theory, we convert the above said problem into deterministic problem. We make use of the fuzzy goal programming technique to obatin the compromise solution of the proposed model as it considerably reduces the calculation in multi level problems as proved by Lachwani[33, 40].

In section 2, we have reviewed the relevant support research done through the available literature. We have presented some definitions and theorems of uncertainty theory which are used in the model in section 3. Notations are given under section 4. In section 5, the mathematical model of uncertain multi level four dimension multi-item fractional transportation problem (UML4DMIFTP) is introduced. Equivalent deterministic models by using expected value method and chance constraint method are given in the sections 5.1 and 5.2 respectively. In section 6 , solution methodology for solving multi level fractional programming is presented. Section 7 contains the procedure for solving the UML4DMIFTP, followed by section 8 consisting of a numerical example and section 9 containing conclusion.

\section{Literature review}

The idea of transportation problem was introduced by Hitchock [1] for dealing with transportation and distribution of goods. In 2018, a new approach for solving multi objective transportation problem (MOTP) was proposed by Lakhveer kaur [2]. Haley [3] in 1962, proposed the solution method for the solid transportation problem(STP) for the first time ever. Bhatia[4], in 1981, presented a solving procedure for obtaining the locally optimum basic feasible solution for STPs with indefinite quadratic objective functions. Charnes and cooper [5] in 1962, proposed a method for conversion of a linear fractional program into linear program. The fractional transportation problem was proposed for the first time by Swarup [6], which was then utilised extensively by many authors like Khurana and Arora [7], Joshi and Gupta [8]. Fuzzy goal programming approach for solving the bi-level linear fractional pogramming, involving a single decision maker at both levels, was proposed by Pramanik and Dey [9] in 2011. A genetic algorithm for solving linear fractional programming problem was proposed by Sameeullah, Devi and Palaniappan [10] in 2008.

Dinkelbach [12] in 1967, developed the solving procedure for multi objective linear fractional programming problem (MOLFPP) which was later employed by Narun Guzel [11] for solving non linear single objective function. Shobana [13] proposed a new algorithm for finding optimal solution in STP, applying in $\alpha$-cut method in uncertain environment. The interactive goal programming method for solving generalised STP was proposed by Acharya et al [14]. The optimal solution for the fully fuzzy multi objective multi item STP was proposed by Deepika Rani and Gulati [15]. Dheyab [16] developed a solving procedure for fuzzy linear fractional programming problem using linear ranking function. Jain [17] introduced a method for solving multi objective fractional programming problem by using the Gauss elimination method. Viwas [18] discussed the more for less paradox suitation which occurs in multi objective fractional transportation problem (MOFTP). A novel approach for solving MOLFPP under fuzzy environment was presented by Moumita and De [19]. To deal with real life situations, uncertainty theory was proposed by Liu [20]. The transportation problem consisting uncertain supply and demand was solved by Guo [21] in his research article. Uncertain linear fractional problem and conversion of optimization problem into equivalent 
Tabel 1. Review on some existing models

\begin{tabular}{|c|c|c|c|c|c|c|c|c|c|}
\hline \multirow{3}{*}{ Reference Article } & \multicolumn{3}{|c|}{ Fractional Objective Function } & \multicolumn{3}{|c|}{ Fuzzy } & \multicolumn{3}{|c|}{ Uncertainty } \\
\hline & \multirow{2}{*}{ Single Objective } & \multicolumn{2}{|c|}{ Multi Objective } & \multicolumn{6}{|c|}{ Dimension } \\
\hline & & Non-hierarchical & Hierarchical & 2D & 3D & 4D & 2D & 3D & 4D \\
\hline Vishwas Deep Joshi [32] & $\sqrt{ }$ & $x$ & $x$ & $x$ & $x$ & $x$ & $x$ & $x$ & $x$ \\
\hline Lachwani [33] & $x$ & $x$ & $\checkmark$ & $x$ & $x$ & $x$ & $x$ & $x$ & $x$ \\
\hline Shiang Taj Liu [34] & $\checkmark$ & $x$ & $x$ & $\checkmark$ & $x$ & $x$ & $x$ & $x$ & $x$ \\
\hline Sheema Sadia [35] & $x$ & $\checkmark$ & $x$ & $\checkmark$ & $x$ & $x$ & $x$ & $x$ & $x$ \\
\hline Syed Aqib Jalil [36] & $x$ & $\checkmark$ & $x$ & $x$ & $x$ & $x$ & $\checkmark$ & $x$ & $x$ \\
\hline Syed Aqib Jalil [31] & $x$ & $x$ & $\checkmark$ & $x$ & $\checkmark$ & $x$ & $x$ & $x$ & $x$ \\
\hline Osman [37] & $x$ & $x$ & $\checkmark$ & $\sqrt{ }$ & $x$ & $x$ & $x$ & $x$ & $x$ \\
\hline Ali Mahmoodirad [23] & $\checkmark$ & $x$ & $x$ & $x$ & $x$ & $x$ & $\checkmark$ & $x$ & $x$ \\
\hline J Merlin Vinotha [38] & $x$ & $\checkmark$ & $x$ & $\checkmark$ & $x$ & $x$ & $x$ & $x$ & $x$ \\
\hline Hamideen Abdei [39] & $x$ & $x$ & $\checkmark$ & $x$ & $\checkmark$ & $x$ & $x$ & $x$ & $x$ \\
\hline Proposed Model & $\checkmark$ & $\checkmark$ & $\checkmark$ & $x$ & $x$ & $x$ & $\checkmark$ & $\checkmark$ & $\checkmark$ \\
\hline
\end{tabular}

crisp problem was proposed by Seyyed Mojtaba [22]. To solve uncertain linear fractional transportation problem, Ali Mahmooderad [23] proposed a model. The multi objective fractional transportation problem under uncertain environment was studied by Revathi and Mohanaselvi [24]. Solution algorithm for solving a multi level programming problem under uncertain conditions was proposed by Liu and Yao [25]. Gao and Kar [26] proposed a method to solve uncertain solid transportation problem involving product blending. To solve fixed charge multi item STP, few uncertain programming models were proposed by Zhang, Peng, Li and Chen [27]. A multi item STP under uncertain environment was studied by Dalman [28]. Cheng, Rao and Chen [29] studied about the multi diemensional Knapsack problem which was based on uncertain measures. The model for uncertain multi objective multi item four dimensional fractional transportation problems was studied by Revathi and Mohanaselvi [30]. The hierarchical decision making model for STP under uncertain conditions was studied by Syed Aqib Jalil, Shakeel Javaid and Syed Mohd Muneeb [31]. Table 1 provides observations based on the existing literature on fractional transportation problems under uncertain environment based on the two dimensions (2D) (Traditional TP i.e. considers only origins and demands), three dimensions (3D) (Solid TP i.e. considers different conveyances along with origins and demands) and four dimensions (4D) (considers multiple items and multiple routes along with 3D). From Table 1, it is clear that there is a gap in terms of developing a ML4DMIFTP in uncertain environment. The most note-worthy aspect is that this study considers uncertainty in all parameters like, origins, demands, transports, items, transportation routes of a transportation problem for the first time.

\section{Preliminaries}

Here, we review some basic definitions and the concepts of uncertainty theory, which will be applied in the subsequent sections.

Definition 3.1: $[20,41]$ Let $\Lambda$ be a $\sigma$-algebra of collection of events $\Lambda$ of a universal set $\Gamma$. A set function $\mathcal{M}$ is said to be uncertain measure defined on the $\sigma$ - algebra where $\mathcal{M}\{\Lambda\}$ indicate the belief degree with which we believe that the event will happens and satisfies the following axioms:

1. Normality Axiom: For the universal set $\Gamma$. We have

$$
\mathcal{M}\{\Gamma\}=1
$$

2. Duality Axiom: For any event $\Lambda$, we have

$$
\mathcal{M}\{\Lambda\}+\mathcal{M}\left\{\Lambda^{c}\right\}=1
$$

3. Subadditivity Axiom: For every countable sequence of events $\Lambda_{1}, \Lambda_{2}, \ldots$ we have

$$
\mathcal{M}\left\{\bigcup_{i=1}^{\infty} \Lambda_{i}\right\} \leq \sum_{i=1}^{\infty} \mathcal{M}\left\{\Lambda_{i}\right\}
$$

4. Product Axiom: Let $\left(\Gamma_{i}, \Lambda_{i}, \mathcal{M}_{i}\right)$ be uncertainty spaces for $i=1,2,3, \ldots$. The 
product uncertain measure is an uncertain measure holds

$$
\mathcal{M}\left\{\prod_{i=1}^{\infty} \Lambda_{i}\right\}=\Lambda_{i=1}^{\infty} \mathcal{M}\left\{\Lambda_{i}\right\}
$$

where $\Lambda_{i} \in \Lambda_{i}$ for $i=1,2, \ldots, \infty$.

Definition 3.2: [20] A function $\xi:(\Gamma, \Lambda, \mathcal{M}) \rightarrow R$ is said to be an uncertain variable such that

$$
\{\xi \in B\}=\{\gamma \in \Gamma / \xi(\gamma) \in B\}
$$

is an event for any Borel set $B$ of real numbers.

Definition 3.3: [20] The uncertainty distribution $\varphi(x)$ of an uncertain variable $\xi$ for any real number $x$ is defined by

$$
\varphi(x)=\mathcal{M}\{\xi \leq \mathrm{x}\}
$$

Definition 3.4: An uncertainty distribution $\varphi(x)$ is said to be regular uncertainty distribution if it is a strictly increasing and continuous function with respect to $x$ at which $0<\varphi(x)<\mathbf{1}$ and

$$
\begin{gathered}
\lim _{x \rightarrow-\infty} \varphi(x)=0 \\
\lim _{x \rightarrow \infty} \varphi(x)=1
\end{gathered}
$$

Definition 3.5: Let $\varphi(x)$ be the regular uncertainty distribution of an uncertain variable $\xi$. Then $\varphi^{-1}(\alpha)$ is called inverse uncertainty distribution of $\xi$ and it exists on $(0,1)$.

Definition 3.6: [20] The uncertain variable $\xi_{i}(i=$ $1,2, \ldots, n)$ are said to be independent if

$$
\mathcal{M}\left\{\bigcap_{i=1}^{n}\left(\xi_{i} \in B_{i}\right)\right\}=\bigwedge_{i=1}^{n} \mathcal{M}\left(\xi_{i} \in B_{i}\right)
$$

where $B_{i}(i=1,2, \ldots, n)$ are called Borel sets of real numbers.

Theorem 3.7: [42] The regular uncertainty distributions of independent uncertain variables $\xi_{i}(i=1,2, \ldots, n)$ are $\phi_{i}(i=1,2, \ldots, n)$ respectively. If the function $f\left(x_{1}, x_{2}, \ldots, x_{n}\right)$ is strictly increasing and strictly decreasing with respect to $x_{1}, x_{2}, \ldots, x_{m}$ and $x_{m+1}, x_{m+2}, \ldots, x_{n}$ respectively then the uncertain variable $\xi=$ $f\left(\xi_{1}, \xi_{2}, \ldots, \xi_{m}, \ldots, \xi_{n}\right)$ has an inverse uncertainty distribution

$$
\begin{gathered}
\psi^{-1}(\alpha)=f\left(\phi_{1}^{-1}(\alpha), \phi_{2}^{-1}(\alpha), \ldots,\right. \\
\phi_{m}^{-1}(\alpha), f\left(\phi_{m+1}^{-1}(1-\alpha),\right. \\
\left.\phi_{m+2}^{-1}(1-\alpha), \ldots, \phi_{n}^{-1}(1-\alpha)\right)
\end{gathered}
$$

Definition 3.8: [20] The expected value of uncertain variable $\xi$ is given by

$$
E(\xi)=\int_{0}^{\infty} \mathcal{M}\{\xi \geq \mathrm{x}\} d x-\int_{-\infty}^{0} \mathcal{M}\{\xi \leq \mathrm{x}\} d x
$$

This is valid only if at least one of the integral is finite.

Theorem 3.9: [43] Let $\phi_{i}(i=1,2, \ldots, n)$ be regular uncertainty distributions of independent $\xi_{i}(i=$ $1,2, \ldots, n)$ with respectively. If the function $f\left(x_{1}, x_{2}, \ldots, x_{n}\right)$ is strictly increasing and strictly decreasing w.r.to $x_{1}, x_{2}, \ldots, x_{m} \quad$ and $x_{m+1}, x_{m+2}, \ldots, x_{n}$ respectively, then

$$
\begin{array}{r}
E(\xi)=\int_{0}^{1} f\left(\phi_{1}^{-1}(\alpha), \ldots, \phi_{m}^{-1}(\alpha), \phi_{m+1}^{-1}(1\right. \\
\left.-\alpha), \ldots, \phi_{n}^{-1}(1-\alpha)\right) d \alpha
\end{array}
$$

From the above theorem, we know that

$$
E(\xi)=\int_{0}^{1} \phi^{-1}(\alpha) d \alpha
$$

Where $\xi$ is an uncertain variable with regular uncertainty distribution $\phi$.

Definition 3.10: [20] The distribution function of a normal uncertain variable is

$$
\phi(x)=\left[1+\exp ^{\left[\frac{\pi(\mu-x)}{\sigma \sqrt{3}}\right]}\right]^{-1}, x \geq 0
$$

and it is denoted as $N(\mu, \sigma) ; \mu, \sigma \in R$ with $\sigma>0$. The inverse uncertainty distribution and the expected value of $\mathrm{N}(\mu, \sigma)$ is defined as follows

$$
\begin{gathered}
\phi^{-1}(\alpha)=\mu+\frac{\sigma \sqrt{3}}{\pi} \ln \frac{\alpha}{1-\alpha} \\
E[\xi]=\mu
\end{gathered}
$$

\section{Nomenclature}

The following notations have been introduced for constructing the proposed UML4DMIFTP model.

\begin{tabular}{|c|c|}
\hline Notation & Definition \\
\hline $\mathrm{S}$ & index for origin, $\mathrm{s}=1,2 . . \mathrm{S}$. \\
\hline $\mathrm{D}$ & index for destination,d=1,2,..D. \\
\hline
\end{tabular}




\begin{tabular}{|c|c|}
\hline V & index of conveyance, $\mathrm{v}=1,2 \ldots \mathrm{V}$ \\
\hline $\mathrm{R}$ & index of route of transportation \\
\hline $\mathrm{W}$ & index of various product \\
\hline$\tilde{Z}^{(n)}$ & $\begin{array}{c}\text { uncertain objective function at } \mathrm{n}^{\text {th }} \text { level, } \\
\text { where } \mathrm{n}=1,2 \ldots . \mathrm{N} \text {. }\end{array}$ \\
\hline$\frac{\tilde{C}_{s d v r w}^{(n)}}{\widetilde{D}_{s d v r w}^{(n)}}$ & $\begin{array}{l}\text { uncertain ratio of unit transportation } \\
\text { actual cost and standard cost of } \mathrm{w}^{\text {th }} \text { good } \\
\text { from } \mathrm{s}^{\text {th }} \text { origin to } \mathrm{d}^{\text {th }} \text { destination by } \mathrm{v}^{\text {th }} \\
\text { transport via } \mathrm{r}^{\text {th }} \text { road per unit distance of } \\
\mathrm{n}^{\text {th }} \text { level objective function. }\end{array}$ \\
\hline$\frac{\tilde{A}_{\text {sdvrw }}^{(n)}}{\tilde{S}_{\text {sdvrw }}^{(n)}}$ & $\begin{array}{l}\text { uncertain ratio of actual transportation } \\
\text { time to the standard transportation time }\end{array}$ \\
\hline$\tilde{a}_{s w}$ & $\begin{array}{c}\text { uncertain quantity of } w^{\text {th }} \text { good available } \\
\text { at } s^{\text {th }} \text { origin }\end{array}$ \\
\hline$\tilde{b}_{d w}$ & $\begin{array}{l}\text { uncertain demand of the } \mathrm{w}^{\text {th }} \text { good at the } \\
\mathrm{d}^{\text {th }} \text { destination }\end{array}$ \\
\hline$\tilde{e}_{v}$ & $\begin{array}{c}\text { uncertain capacity of a single } \mathrm{v}^{\text {th }} \text { type } \\
\text { transport }\end{array}$ \\
\hline$d_{N^{+}}^{(n)}, d_{D^{+}}^{(n)}$ & $\begin{array}{l}\text { positive deviational variable of the } \mathrm{n}^{\text {th }} \\
\text { level objective's numerator and } \\
\text { denominator respectively. }\end{array}$ \\
\hline$d_{N^{-}}^{(n)}, d_{D^{-}}^{(n)}$ & $\begin{array}{l}\text { negative deviational variable of the } \mathrm{n}^{\text {th }} \\
\text { level objective's numerator and } \\
\text { denominator respectively. }\end{array}$ \\
\hline$d_{+}^{(n)}, d_{-}^{(n)}$ & $\begin{array}{l}\text { positive and negative deviational } \\
\text { variables for } \mathrm{n}^{\text {th }} \text { level decision vectors. }\end{array}$ \\
\hline
\end{tabular}

\section{Mathematical formulation}

The proposed model for an uncertain multi level four diemension multi-item fractional transportation problem [UML4DMIFTP] is presented below. The proposed model is created by considering w products to be transported from $\mathrm{S}$ origins to $\mathrm{D}$ destinations by means of $\mathrm{v}$ conveyances via $\mathrm{r}^{\text {th }}$ route and objectives are to be minimized in all $n$ levels, where $n=1,2, \ldots N$. The general form of UML4DMIFTP is given in (10). where $\bar{X}^{n}=\left\{X_{1}^{n}, X_{2}^{n}, \ldots, X_{M_{n}}^{n}\right\}$, decision variables under the control of $\mathrm{n}^{\text {th }}$ level decision maker. Here, $\bar{X}=\bar{X}^{1} U \bar{X}^{2} U \bar{X}^{3}, \ldots, \bar{X}^{N}$. Due to the uncertainty that exists in the parameters, it cannot be optimized directly. An equivalent deterministic model for the proposed model is created by using the expected value and chance constraint model based on uncertainty theory for obtaining the compromise solution. In this proposed model, the parameters are considered as the normal uncertain variables.

Level 1

$$
\begin{aligned}
& \operatorname{Min}_{\bar{X}^{1}} \tilde{Z}^{(1)} \\
& =\frac{\sum_{s=1}^{S} \sum_{d=1}^{D} \sum_{v=1}^{V} \sum_{r=1}^{R} \sum_{w=1}^{W} \tilde{C}_{s d v r w}^{(1)} x_{s d v r w}}{\sum_{s=1}^{S} \sum_{d=1}^{D} \sum_{v=1}^{V} \sum_{r=1}^{R} \sum_{w=1}^{W} \widetilde{D}_{s d v r w}^{(1)} x_{s d v r w}}
\end{aligned}
$$

Level 2

$$
\begin{aligned}
& \operatorname{Min}_{\bar{X}^{2}} \tilde{Z}^{(2)} \\
& =\frac{\sum_{s=1}^{S} \sum_{d=1}^{D} \sum_{v=1}^{V} \sum_{r=1}^{R} \sum_{w=1}^{W} \tilde{C}_{s d v r w}^{(2)} x_{s d v r w}}{\sum_{s=1}^{S} \sum_{d=1}^{D} \sum_{v=1}^{V} \sum_{r=1}^{R} \sum_{w=1}^{W} \widetilde{D}_{s d v r w}^{(2)} x_{s d v r w}} \\
& \vdots
\end{aligned}
$$

Level $n$

$$
\begin{aligned}
& \operatorname{Min}_{\bar{X}^{n}} \tilde{Z}^{(n)} \\
& =\frac{\sum_{s=1}^{S} \sum_{d=1}^{D} \sum_{v=1}^{V} \sum_{r=1}^{R} \sum_{w=1}^{W} \tilde{C}_{s d v r w}^{(n)} x_{s d v r w}}{\sum_{s=1}^{S} \sum_{d=1}^{D} \sum_{v=1}^{V} \sum_{r=1}^{R} \sum_{w=1}^{W} \widetilde{D}_{\text {sdvrw }}^{(n)} x_{s d v r w}}
\end{aligned}
$$

Subject to

$$
\begin{gathered}
\sum_{d=1}^{D} \sum_{v=1}^{V} \sum_{r=1}^{R} x_{s d v r w} \leq \tilde{a}_{s w} \quad s=1,2, \ldots, S, w \\
=1,2, \ldots, W \\
\sum_{s=1}^{S} \sum_{v=1}^{V} \sum_{r=1}^{R} x_{\text {sdvrw }} \leq \tilde{b}_{d w} \quad d=1,2, \ldots, D, w \\
\sum_{S=1}^{S} \sum_{d=1}^{D} \sum_{r=1}^{R} \sum_{w=1}^{W} x_{s d v r w} \leq \tilde{e}_{v} \quad v=1,2, \ldots, W, V \\
x_{\text {sdvrw }} \geq 0 \forall s, d, v, r, w
\end{gathered}
$$

\subsection{Expected value model}

An equivalent deterministic model for UML4DMIFTP has been presented in this section. By using the expected value method for normal uncertain variable and its properties, the equivalent deterministic model for UML4DMIFTP is given in Eq. (18).

Level 1

$$
\begin{aligned}
& \operatorname{Min}_{\bar{X}^{1}} Z^{(1)} \\
& =\frac{\sum_{s=1}^{S} \sum_{d=1}^{D} \sum_{v=1}^{V} \sum_{r=1}^{R} \sum_{w=1}^{W} E\left(\tilde{C}_{s d v r w}^{(1)}\right) x_{s d v r w}}{\sum_{s=1}^{S} \sum_{d=1}^{D} \sum_{v=1}^{V} \sum_{r=1}^{R} \sum_{w=1}^{W} E\left(\widetilde{D}_{\text {sdvrw }}^{(1)}\right) x_{s d v r w}}
\end{aligned}
$$

Level 2

$$
\begin{aligned}
& \operatorname{Min}_{\bar{X}^{2}} Z^{(2)} \\
& =\frac{\sum_{s=1}^{S} \sum_{d=1}^{D} \sum_{v=1}^{V} \sum_{r=1}^{R} \sum_{w=1}^{W} E\left(\tilde{C}_{s d v r w}^{(2)}\right) x_{s d v r w}}{\sum_{s=1}^{S} \sum_{d=1}^{D} \sum_{v=1}^{V} \sum_{r=1}^{R} \sum_{w=1}^{W} E\left(\widetilde{D}_{\text {sdvr }}^{(2)}\right) x_{\text {sdvrw }}}
\end{aligned}
$$

Level $\mathrm{n}$

$$
\begin{aligned}
& \operatorname{Min}_{X^{n}} Z^{(n)} \\
& =\frac{\sum_{s=1}^{S} \sum_{d=1}^{D} \sum_{v=1}^{V} \sum_{r=1}^{R} \sum_{w=1}^{W} E\left(\tilde{C}_{s d v r w}^{(n)}\right) x_{s d v r w}}{\sum_{s=1}^{S} \sum_{d=1}^{D} \sum_{v=1}^{V} \sum_{r=1}^{R} \sum_{w=1}^{W} E\left(\widetilde{D}_{\text {sdvrw }}^{(n)}\right) x_{s d v r w}}
\end{aligned}
$$


Subject to

$$
\begin{gathered}
\sum_{d=1}^{D} \sum_{v=1}^{V} \sum_{r=1}^{R} x_{s d v r w}-E\left(\tilde{a}_{s w}\right) \leq 0 \quad s=1,2, \ldots, S, w \\
=1,2, \ldots, W \\
E\left(\tilde{b}_{d w}\right)-\sum_{s=1}^{S} \sum_{v=1}^{V} \sum_{r=1}^{R} x_{s d v r w} \leq 0 \quad d \\
=1,2, \ldots, D, w=1,2, \ldots, W
\end{gathered}
$$

$$
\begin{gathered}
\sum_{s=1}^{S} \sum_{d=1}^{D} \sum_{r=1}^{R} \sum_{w=1}^{W} x_{s d v r w}-E\left(\tilde{e}_{v}\right) \leq 0 v=1,2, \ldots, V \\
x_{s d v r w} \geq 0 \forall s, d, v, r, w
\end{gathered}
$$

\subsection{Chance constrained}

An equivalent deterministic model for an UML4DMIFTP by using chance constraint method is presented in this section.

Level 1

$$
\operatorname{Min}_{\bar{X}^{1}} \widetilde{Z}^{(1)}=\frac{\sum_{s=1}^{S} \sum_{d=1}^{D} \sum_{v=1}^{V} \sum_{r=1}^{R} \sum_{w=1}^{W}\left(\chi^{(1)}\right)_{s d v r w}^{-1}\left(\alpha_{1}\right) x_{s d v r w}}{\sum_{s=1}^{S} \sum_{d=1}^{D} \sum_{v=1}^{V} \sum_{r=1}^{R} \sum_{w=1}^{W}\left(\phi^{(1)}\right)_{s d v r w}^{-1}\left(\gamma_{1}\right) x_{s d v r w}}
$$

Level 2

$$
\operatorname{Min}_{\bar{X}^{2}} \tilde{Z}^{(2)}=\frac{\sum_{s=1}^{S} \sum_{d=1}^{D} \sum_{v=1}^{V} \sum_{r=1}^{R} \sum_{w=1}^{W}\left(\chi^{(2)}\right)_{s d v r w}^{-1}\left(\alpha_{2}\right) x_{s d v r w}}{\sum_{s=1}^{S} \sum_{d=1}^{D} \sum_{v=1}^{V} \sum_{r=1}^{R} \sum_{w=1}^{W}\left(\phi^{(2)}\right)_{s d v r w}^{-1}\left(\gamma_{2}\right) x_{s d v r w}}
$$

Level $n$

$$
\operatorname{Min}_{\bar{X}^{n}} \tilde{Z}^{(n)}=\frac{\sum_{s=1}^{S} \sum_{d=1}^{D} \sum_{v=1}^{V} \sum_{r=1}^{R} \sum_{w=1}^{W}\left(\chi^{(n)}\right)_{s d v r w}^{-1}\left(\alpha_{n}\right) x_{s d v r w}}{\sum_{s=1}^{S} \sum_{d=1}^{D} \sum_{v=1}^{V} \sum_{r=1}^{R} \sum_{w=1}^{W}\left(\phi^{(n)}\right)_{s d v r w}^{-1}\left(\gamma_{n}\right) x_{s d v r w}}
$$

Subject to

$$
\begin{gathered}
\sum_{d=1}^{D} \sum_{v=1}^{V} \sum_{r=1}^{R} x_{s d v r w} \leq \psi_{s w}^{-1}\left(1-\alpha_{s w}\right) \quad s=1,2, \ldots, S, w=1,2, \ldots, W \\
\sum_{S=1}^{S} \sum_{v=1}^{V} \sum_{r=1}^{R} x_{s d v r w} \geq \theta_{d w}^{-1}\left(\beta_{d w}\right) \quad d=1,2, \ldots, D, w=1,2, \ldots, W \\
\sum_{s=1}^{S} \sum_{d=1}^{D} \sum_{r=1}^{R} \sum_{w=1}^{W} x_{s d v r w} \leq \lambda_{v}^{-1}\left(1-\beta_{v}\right) \quad v=1,2, \ldots, V \\
x_{s d v r w} \geq 0 \forall s, d, v, r, w
\end{gathered}
$$

Suppose that $\quad \widetilde{D}_{\text {sdvrw }}^{(n)}(n=$ $1,2, \ldots, N), \tilde{C}_{s d v r w}^{(n)}(n=1,2, \ldots, N), \tilde{a}_{s w}, \tilde{b}_{d w}, \tilde{e}_{v}$, are independent uncertain variables with regular uncertain

$\chi_{s d v r w}^{(n)}, \phi_{s d v r w}^{(n)}, \psi_{s w}, \theta_{d w}, \lambda_{v},(n=1,2, \ldots, N$

respectively. The proposed UML4DMIFTP's equivalent deterministic model using the chance constrained method is given in Eq. (19) where $\alpha_{n}, \gamma_{n}, \beta_{v}, \alpha_{s w}$ and $\beta_{d w} \forall n, v, s, w \& d$ are predetermined confidence levels and $\alpha_{n}, \gamma_{n}, \beta_{v}, \alpha_{s w}$ and $\beta_{d w} \in(0,1), \forall n, v, s, w \& d$. By applying the properties of chance constraint method for normal uncertain variable, an equivalent model of Eq. (19) is given in Eq. (20).

Definition 5.3 A point is said to be an efficient solution of UML4DMIFTP iff there does not exist another $x \in X$ s.t. $Z_{n}(x) \leq Z_{n}\left(x^{0}\right)$, and $Z_{n}(x)<$ $Z_{n}\left(x^{0}\right)$, for at least one $\mathrm{n}$.

\section{Solution methodology}

When more than one goal is present, to obtain the satisfactory solution the goal programming technique was proposed by Charnes Clan Cooper [44]. The goal programming technique was further developed by T.Chang [45], Pal [46] etc. To solve multi objective transportation problem (MOTP), a new fuzzy goal programming technique was introduced by Mohammed [47], which was later used by Zangiabadi $[48,49]$ to solve MOTP containing linear as well as non-linear membership functions. The main aim of goal programming (GP) is to minimize the distance between $\mathrm{Z}$ and aspiration (or) target level $\bar{Z}$. The 
positive and negative deviational variables are defined as in Eq. (21) and Eq. (22).

Level 1

$$
\operatorname{Min}_{\bar{X}^{1}} \widetilde{Z}^{(1)}=\frac{\sum_{s=1}^{S} \sum_{d=1}^{D} \sum_{v=1}^{V} \sum_{r=1}^{R} \sum_{w=1}^{W}\left(e_{s d v r w}^{1}+\frac{\sigma_{s d v r w}^{1}}{\pi} \sqrt{3} \ln \frac{\alpha_{1}}{\left(1-\alpha_{1}\right)}\right) x_{s d v r w}}{\sum_{s=1}^{S} \sum_{d=1}^{D} \sum_{v=1}^{V} \sum_{r=1}^{R} \sum_{w=1}^{W}\left(e_{s d v r w}^{1}+\frac{\sigma_{s d v r w}^{1}}{\pi} \sqrt{3} \ln \frac{\gamma_{1}}{\left(1-\gamma_{1}\right)}\right) x_{s d v r w}}
$$

Level 2

$$
\operatorname{Min}_{\bar{X}^{2}} \tilde{Z}^{(2)}=\frac{\sum_{s=1}^{S} \sum_{d=1}^{D} \sum_{v=1}^{V} \sum_{r=1}^{R} \sum_{w=1}^{W}\left(e_{s d v r w}^{2}+\frac{\sigma_{s d v r w}^{2}}{\pi} \sqrt{3} \ln \frac{\alpha_{2}}{\left(1-\alpha_{2}\right)}\right) x_{s d v r w}}{\sum_{s=1}^{S} \sum_{d=1}^{D} \sum_{v=1}^{V} \sum_{r=1}^{R} \sum_{w=1}^{W}\left(e_{s d v r w}^{2}+\frac{\sigma_{s d v r w}^{2}}{\pi} \sqrt{3} \ln \frac{\gamma_{2}}{\left(1-\gamma_{2}\right)}\right) x_{s d v r w}}
$$

Level $n$

$$
\operatorname{Min}_{\bar{X}^{n}} \tilde{Z}^{(n)}=\frac{\sum_{s=1}^{S} \sum_{d=1}^{D} \sum_{v=1}^{V} \sum_{r=1}^{R} \sum_{w=1}^{W}\left(e_{s d v r w}^{n}+\frac{\sigma_{s d v r w}^{n}}{\pi} \sqrt{3} \ln \frac{\alpha_{n}}{\left(1-\alpha_{n}\right)}\right) x_{s d v r w}}{\sum_{S=1}^{S} \sum_{d=1}^{D} \sum_{v=1}^{V} \sum_{r=1}^{R} \sum_{w=1}^{W}\left(e_{s d v r w}^{n}+\frac{\sigma_{s d v r w}^{n}}{\pi} \sqrt{3} \ln \frac{\gamma_{n}}{\left(1-\gamma_{n}\right)}\right) x_{s d v r w}}
$$

Subject to

$$
\begin{gathered}
\sum_{d=1}^{D} \sum_{v=1}^{V} \sum_{r=1}^{R} x \leq e_{s w}+\frac{\sigma_{s w} \sqrt{3}}{\pi} \ln \frac{1-\alpha_{s w}}{\alpha_{s w}}, \quad s=1,2, \ldots, S, w=1,2, \ldots, W \\
\sum_{s=1}^{S} \sum_{v=1}^{V} \sum_{r=1}^{R} x \geq e_{d w}+\frac{\sigma_{d w} \sqrt{3}}{\pi} \ln \frac{\beta_{d w}}{1-\beta_{d w}}, \quad d=1,2, \ldots, D, w=1,2, \ldots, W \\
\sum_{s=1}^{S} \sum_{d=1}^{D} \sum_{r=1}^{R} \sum_{w=1}^{W} x_{s d v r w} \leq e_{v}+\frac{\sigma_{v} \sqrt{3}}{\pi} \ln \frac{1-\beta_{v}}{\beta_{v}}, v=1,2, \ldots, V \\
x_{s d v r w} \geq 0 \forall s, d, v, r, w
\end{gathered}
$$$$
D_{n}^{+}=\max \left(0, Z_{n}-\bar{Z}_{n}\right)
$$$$
D_{n}^{-}=\max \left(0, \bar{Z}_{n}-Z_{n}\right)
$$

When the aim is to maximize $Z_{n}$, we obtain the optimal solution by minimizing the negative deviational variable. Similarly, when the aim is to minimize $Z_{n}$, we obtain the optimal solution by minimizing the positive deviational variable. When we desire $Z_{n}=\bar{Z}_{n}$, we obtain the optimal solution by minimizing $D_{n}^{+}+D_{n}^{-}$. To formulate membership functions the fuzzy goals and their aspiration levels has to be defined first. Firstly, we maximize and minimize the numerator and denominator objective functions individually for each level of decision making. After finding the maximum and minimum values of each objective function, we construct the payoff matrices. Each row's maximum values $\bar{N}^{(n)}$ and $\bar{D}^{(n)} \forall n=1,2, \ldots, N$ are known as the aspired level or upper tolerance limit for the membership function of $\mathrm{n}^{\text {th }}$ level numerator and denominator objectives respectively. Likewise, the minimum values of each row $\underline{N}^{(n)}$ and $\underline{D}^{(n)} \forall n=1,2, \ldots, N$ are lower tolerance limit for the membership function of the $\mathrm{n}^{\text {th }}$ level numerator and denominator respectively. 
The linear membership functions for fuzzy goals are defined as follows:

$$
\begin{gathered}
\mu\left(N^{(n)}(\bar{X})\right)=\left\{\begin{array}{clc}
1 & \text { if } & N^{(n)}(\bar{X}) \leq \underline{N}^{(n)} \\
\frac{\bar{N}^{(n)}-N^{(n)}(\bar{X})}{\bar{N}^{(n)}-\underline{N}^{(n)}} & \text { if } & \underline{N}^{(n)} \leq N^{(n)}(\bar{X}) \leq \bar{N}^{(n)}, \forall n=1,2, \ldots, N \\
0 & \text { if } & \underline{N}^{(n)}(\bar{X}) \geq \bar{N}^{(n)}
\end{array}\right. \\
\mu\left(D^{(n)}(\bar{X})\right)=\left\{\begin{array}{ccc}
0 & \text { if } & D^{(n)}(\bar{X}) \leq \underline{D}^{(n)} \\
\frac{\bar{D}^{(n)}(\bar{X})-\underline{D}^{(n)}}{\bar{D}^{(n)}-\underline{D}^{(n)}} & \text { if } & \underline{D}^{(n)} \leq D^{(n)}(\bar{X}) \leq \bar{D}^{(n)}, \forall n=1,2, \ldots, N \\
1 & \text { if } & \underline{D}^{(n)}(\bar{X}) \geq \bar{D}^{(n)}
\end{array}\right.
\end{gathered}
$$

Comparably, the decision vector $\mathrm{X}^{\mathrm{n}}$ 's membership function as follows, where $(\mathrm{n}=1,2, \ldots \mathrm{N})$.

$$
\mu\left(X^{n}\right)=\left\{\begin{array}{ccc}
1 & \text { if } & X^{n} \leq \underline{X} \\
\frac{\bar{X}-\left(X^{n}\right)}{\bar{X}-\underline{X}} & \text { if } & \underline{X} \leq\left(X^{n}\right) \leq \bar{X}, \forall n=1,2, \ldots, N \\
0 & \text { if } & X^{n} \geq \bar{X}
\end{array}\right.
$$

where $\bar{X}^{n}$ and $\underline{X}^{n}$ are represents the values of corresponding decision vectors at each level which yield the maximum and minimum values of the numerator part of the objective functions $\bar{N}^{n}(\bar{X})$ and $\underline{N}^{n}(\underline{X}), \forall n=1,2, \ldots, N-1$ at every level respectively is given by:

$$
\begin{gathered}
\bar{X}^{n}=\operatorname{Max}_{\bar{X}^{n} \in X}\left\{\bar{N}^{(n)}(\bar{X}), \forall n=1,2, \ldots, N\right\} \\
\underline{X}^{n}=\operatorname{Min}_{\bar{X}^{n} \in X}\left\{\underline{N}^{n}(\bar{X}), \forall n=1,2, \ldots, N\right\}
\end{gathered}
$$

$\min \delta=\sum_{n=1}^{N} d_{N^{+}}^{(n)}+\sum_{n=1}^{N} d_{D^{+}}^{(n)}+\sum_{n=1}^{N-1} d_{+}^{(n)}$

Subject to

$$
\begin{gathered}
\mu\left(N^{(n)}\right)+d_{N^{-}}^{(n)}-d_{N^{+}}^{(n)}=1, \forall n=1,2, \ldots, N \\
\mu\left(D^{(n)}\right)+d_{D^{-}}^{(n)}-d_{D^{+}}^{(n)}=1, \forall n=1,2, \ldots, N \\
\mu\left(X^{(n)}\right)+d_{-}^{(n)}-d_{+}^{(n)}=1, \forall n=1,2, \ldots, N \\
\sum_{d=1}^{D} \sum_{v=1}^{V} \sum_{r=1}^{R} x_{s d v r w}-E\left(\tilde{a}_{s w}\right) \leq 0 \quad s=1,2, \ldots, S, w \\
=1,2, \ldots, W \\
E\left(\tilde{b}_{d w}\right)-\sum_{s=1}^{S} \sum_{v=1}^{V} \sum_{r=1}^{R} x_{s d v r w} \leq 0 \\
=1,2, \ldots, D, w=1,2, \ldots, W
\end{gathered}
$$

$$
\begin{gathered}
\sum_{s=1}^{S} \sum_{d=1}^{D} \sum_{r=1}^{R} \sum_{w=1}^{W} x_{s d v r w}-E\left(\tilde{e}_{v}\right) \leq 0 \quad v \\
=1,2, \ldots, V \\
x_{\text {sdvrw }} \geq 0 \forall s, d, v, r, w
\end{gathered}
$$

Since, the objective functions generally conflict each other, the completely satisfactory optimal solution is very rarely obtained; the highest degree of membership value for each fuzzy goal can be 1 . So, we need to minimize the regret of each decision maker at all levels and every decision maker should try to maximize the membership function by reducing the distance between membership value and unity and minimize the positive deviational value. In this process all objective functions are simultaneously optimized. The model UML4DMIFTP (18) is written as (27). Therefore, we can note that only the sum of over deviational variables has to be minimized to reach the aspiration level. When the aspired level is reached, the negative deviational value is zero. When the achievement level is zero, negative deviational value becomes unity. UML4DMIFTP (27) becomes as follows:

$\min \delta=\sum_{n=1}^{N} d_{N^{+}}^{(n)}+\sum_{n=1}^{N} d_{D^{+}}^{(n)}+\sum_{n=1}^{N-1} d_{+}^{(n)}$ 
Subject to

$$
\begin{aligned}
& \underline{N}^{(n)}-N^{(n)}(\bar{X})+d_{N^{-}}^{(n)}\left(\bar{N}^{(n)}-\underline{N}^{(n)}\right) \geq 0, \forall n \\
& =1,2, \ldots, N \\
& -\bar{D}^{(n)}+D^{(n)}(\bar{X})+d_{N^{-}}^{(n)}\left(\bar{D}^{(n)}-\underline{D}^{(n)}\right) \geq 0, \forall n \\
& =1,2, \ldots, N \\
& \underline{X}-X^{(n)}+d_{-}^{(n)}(\bar{X}-\underline{X}) \geq 0, \forall n=1,2, \ldots, N-1 \\
& \sum_{d=1}^{D} \sum_{v=1}^{V} \sum_{r=1}^{R} x_{s d v r w}-E\left(\tilde{a}_{s w}\right) \leq 0 \quad s=1,2, \ldots, S, w \\
& =1,2, \ldots, W \\
& E\left(\tilde{b}_{d w}\right)-\sum_{S=1}^{S} \sum_{v=1}^{V} \sum_{r=1}^{R} x_{s d v r w} \leq 0 d \\
& =1,2, \ldots, D, w=1,2, \ldots, W \\
& \sum_{S=1}^{S} \sum_{d=1}^{D} \sum_{r=1}^{R} \sum_{w=1}^{W} x_{s d v r w}-E\left(\tilde{e}_{v}\right) \leq 0, v=1,2, \ldots, V \\
& x_{s d v r w} \geq 0 \forall s, d, v, r, w
\end{aligned}
$$

\section{Solution procedure for UML4DMIFTP}

Step 1: Formulate the decision making model for uncertain multi level four dimensional multi item fractional transportation problem as in Eq. (17).

Step 2: Obtain an equivalent deterministic model for UML4DMIFTP by using expected value model and chance constraint model on uncertainty theory as in Eq. (18) and Eq. (20).

Step 3: Under the given constraints, for all objectives, calculate the individual max $\left(\bar{N}^{(n)}\right.$ and $\left.\bar{D}^{(n)}\right)$ and $\min \left(\underline{N}^{(n)}\right.$ and $\left.\underline{D}^{(n)}\right)$ values of numerator and denominator for all levels respectively.

Step 4: For all levels and all objectives, set the fuzzy goals and aspiration levels $\underline{N}^{(n)}, \underline{N}^{(n)}$ (or) $\bar{D}^{(n)}, \underline{D}^{(n)}$ for each and every numerator and denominator parts. Step 5: Compute the highest and lowest value of numerator part of all objectives respectively as defined in Eq. (26).

Step 6: Set corresponding values of decision variables as aspiration levels for membership functions of the vector $X^{(n)}, \forall n=1,2, \ldots, N-1$.

Step 7: Find the membership functions of numerators $\mu\left(N^{(n)}\right)$, denominators $\mu\left(D^{(n)}\right)$ and decision variables $\mu\left(X^{(n)}\right)$.
Step 8: For the proposed UML4DMIFTP, formulate the fuzzy goal programming model as in Eq (28).

Step 9: Using generalized reduced gradient technique (LINGO-18.0 Suite Solver), solve the fuzzy goal programming model to have the compromise solution of proposed UML4DMIFTP.

\section{Numerical example}

When the problem analysed in the work aims of formulating an optimal transportation scheme considering real life objectives like minimisation of actual cost and standard cost ratio, minimizing time and breakability factor of product shipping. The problem considers a hierarchical decision making system wherein the higher level decision maker deals with minimizing the actual cost-standard cost ratio. The second level decision maker is associated with minimizing the time while the third level decision maker is interest on mimimizing the defective items which would be rejected. İn the situation considered in this paper, there are two items, origins, destinations, conveyances each. All the parameters involved are taken as normal variables as the data available for the problem is continous. The data for the availabilities in various sources,demands of the destinations and capacities of transports are presented in table 2 , table 3 and table 4 respectively.

Table 5 contains the data for ratio between the actual and standard transportation cost, ratio between the actual and standard transportation time and ratio between actual and standard demage items. For the formulation of the problem and getting the compromise solution, we may follow the following steps:

Step 1: The decision making model is formulated for UML4DMIFTP for above data as of Eq. (17).

Step 2: We convert the above UML4DMIFTP model into deterministic model by making use of expected value method on uncertainity theory as Eq. (18).

Step 3: Calculate the individual max $\left(\bar{N}^{(n)}, \bar{D}^{(n)}\right)$ and $\min \left(N^{(n)}, \underline{D}^{(n)}\right)$ for all levels under the given constraints. The optimal values of each numerator and denominator have been presented in table (6).

Step 4: Using the steps 4-8 given in section 7, formulate the fuzzy goal programming model for UML4DMIFTP as in Eq. (28).

Step 5: The problem obtained in step 8 has been solved using the reduced gradient technique to obtain the compromise solution of the proposed UML4DMIFTP problem. The compromise solution for the proposed UML4DMIFTP is: 
Table 2. Availabilities in origin

\begin{tabular}{|c|c|c|}
\hline $\mathbf{S}$ & $\mathbf{w}$ & $\tilde{a}_{s w}$ \\
\hline & 1 & $(200,10)$ \\
\cline { 2 - 3 } 1 & 2 & $(360,20)$ \\
\hline 2 & 1 & $(225,30)$ \\
\hline
\end{tabular}

Table 3. Demands in destination

\begin{tabular}{|c|c|c|}
\hline $\mathbf{d}$ & $\mathbf{w}$ & $\tilde{b}_{d w}$ \\
\hline & 1 & $(150,5)$ \\
\cline { 2 - 3 } 1 & 2 & $(125,10)$ \\
\hline 2 & 1 & $(200,15)$ \\
\hline
\end{tabular}

Table 4. Capacity of conveyance

\begin{tabular}{|c|c|c|}
\hline $\mathbf{v}$ & $\mathbf{r}$ & $\tilde{e}_{v r}$ \\
\hline \multirow{2}{*}{1} & 1 & $(280,15)$ \\
\cline { 2 - 3 } & 2 & $(250,20)$ \\
\hline \multirow{2}{*}{2} & 1 & $(290,25)$ \\
\cline { 2 - 3 } & 2 & $(380,10)$ \\
\hline
\end{tabular}

Table 5. Ratios of actual and standard unit transportation cost, actual and standard transportation time, actual and standard damaged items

\begin{tabular}{|c|c|c|c|c|c|c|c|c|c|}
\hline \multirow{3}{*}{$\mathrm{s}$} & \multirow{3}{*}{$\mathrm{d}$} & \multirow{3}{*}{$\mathrm{v}$} & \multirow{3}{*}{$\mathrm{r}$} & \multicolumn{2}{|c|}{ Level 1} & \multicolumn{2}{|c|}{ Level 2} & \multicolumn{2}{|c|}{ Level 3} \\
\hline & & & & \multicolumn{2}{|c|}{$\begin{array}{l}\text { Ratio of actual Unit cost } \\
\text { and standard unit cost }\end{array}$} & \multicolumn{2}{|c|}{$\begin{array}{l}\text { Ratio of actual and standard } \\
\text { transportation time }\end{array}$} & \multicolumn{2}{|c|}{$\begin{array}{c}\text { Ratio of actual and } \\
\text { standard damage items }\end{array}$} \\
\hline & & & & $\frac{\tilde{C}_{s d v r 1}^{(1)}}{\widetilde{D}_{s d v r 1}^{(1)}}$ & $\frac{\tilde{C}_{s d v r 2}^{(1)}}{\widetilde{D}_{s d v r 2}^{(1)}}$ & $\frac{\tilde{A}_{s d v r 1}^{(2)}}{\tilde{S}_{s d v r 1}^{(2)}}$ & $\frac{\tilde{A}_{s d v r 2}^{(2)}}{\tilde{S}_{s d v r 2}^{(2)}}$ & $\frac{\tilde{C}_{\text {sdvr } 1}^{(3)}}{\widetilde{D}_{\text {sdvr } 1}^{(3)}}$ & $\frac{\tilde{C}_{s d v r 2}^{(3)}}{\widetilde{D}_{s d v r 2}^{(3)}}$ \\
\hline \multirow{16}{*}{1} & \multirow{8}{*}{1} & \multirow{4}{*}{1} & 1 & $(28,1)$ & $(32,2)$ & $(28,0.5)$ & $(12,3)$ & $(19,1.5)$ & $(19,2)$ \\
\hline & & & & $\overline{(38,2)}$ & $\overline{(32,3)}$ & $\overline{(18,2)}$ & $\overline{(27,1)}$ & $\overline{(21,2)}$ & $\overline{(16,3)}$ \\
\hline & & & & $(28,1.5)$ & $(38,2)$ & $(28,3)$ & $(12,3)$ & $(19,2)$ & $(9,3)$ \\
\hline & & & 2 & $\overline{(38,1.8)}$ & $\overline{(38,2.5)}$ & $\overline{(18,2)}$ & $\overline{(24,1.5)}$ & $(36,2.5)$ & $\overline{(26,3.5)}$ \\
\hline & & \multirow{4}{*}{2} & & $(29,3)$ & $(39,4)$ & $(12,2.5)$ & $(21,1.5)$ & $(9,1)$ & $(9,2)$ \\
\hline & & & 1 & $\overline{(29,2)}$ & $\overline{(39,3)}$ & $\overline{(19,3)}$ & $\overline{(24,3.5)}$ & $\overline{(21,1.5)}$ & $\overline{(36,2.5)}$ \\
\hline & & & & $(29,2)$ & $(29,3)$ & $(18,2)$ & $(18,2)$ & $(29,2)$ & $(39,2.5)$ \\
\hline & & & 2 & $\overline{(29,1.5)}$ & $\overline{(29,2.5)}$ & $(19,3)$ & $(19,2)$ & $(36,3)$ & $(16,3.5)$ \\
\hline & \multirow{8}{*}{2} & \multirow{4}{*}{1} & & $(27,1)$ & $(39,1.5)$ & $(18,1)$ & $(21,2.5)$ & $(29,4)$ & $(49,2.5)$ \\
\hline & & & 1 & $\overline{(17,2)}$ & $\overline{(39,3)}$ & $\overline{(18,1)}$ & $\overline{(37,1.5)}$ & $\overline{(22,3)}$ & $\overline{(38,1.5)}$ \\
\hline & & & & $(39,2)$ & $(39,3)$ & $(16,2)$ & $(27,1.5)$ & $(39,1)$ & $(39,2)$ \\
\hline & & & 2 & $\overline{(39,4)}$ & $\overline{(39,4.5)}$ & $\overline{(37,2.5)}$ & $(37,3)$ & $\overline{(22,2)}$ & $\overline{(22,2)}$ \\
\hline & & \multirow{4}{*}{2} & & $(29,2)$ & $(34,1.5)$ & $(16,1.5)$ & $(21,2)$ & $(39,3)$ & $(11,5)$ \\
\hline & & & 1 & $\overline{(29,1.5)}$ & $\overline{(30,2)}$ & $\overline{(18,1)}$ & $\overline{(21,0.5)}$ & $\overline{(42,4)}$ & $\overline{(31,4)}$ \\
\hline & & & & $(39,2.5)$ & $(34,3.5)$ & $(16,2)$ & $(21,2.5)$ & $(6,2.5)$ & $(11,3.5)$ \\
\hline & & & 2 & $\overline{(49,0.5)}$ & $\overline{(34,0.8)}$ & $\overline{(28,4)}$ & $(23,3.5)$ & $(41,3)$ & $\overline{(41,4)}$ \\
\hline \multirow{16}{*}{2} & \multirow{8}{*}{1} & \multirow{4}{*}{1} & & $(39,4)$ & $(38,4.5)$ & $(28,1.5)$ & $(21,1.5)$ & $(11,2)$ & $(11,1.5)$ \\
\hline & & & 1 & $\overline{(39,4)}$ & $(48,4)$ & $(18,8)$ & $(28,2)$ & $(22,2)$ & $(41,2)$ \\
\hline & & & & $(39,1.5)$ & $(39,3)$ & $(18,2)$ & $(18,1.5)$ & $(26,1)$ & $(26,1.5)$ \\
\hline & & & 2 & $(39,2)$ & $(39,2.5)$ & $(18,3)$ & $(18,4)$ & $(41,2)$ & $\overline{(21,2)}$ \\
\hline & & \multirow{4}{*}{2} & & $(31,2)$ & $(34,2.5)$ & $(18,1.5)$ & $(22,1)$ & $(16,3)$ & $(31,2)$ \\
\hline & & & 1 & $(31,4)$ & $(34,3)$ & $(18,5)$ & $(25,0.5)$ & $(17,1.5)$ & $(23,2)$ \\
\hline & & & & $(34,3)$ & $(34,3.5)$ & $(28,2)$ & $(22,3)$ & $(26,2)$ & $(26,4)$ \\
\hline & & & 2 & $(34,2)$ & $(34,1.5)$ & $(25,3)$ & $(25,2.5)$ & $(17,3)$ & $(17,5)$ \\
\hline & \multirow{8}{*}{2} & \multirow{4}{*}{1} & & $(38,2)$ & $(35,4)$ & $(28,3)$ & $(22,1.5)$ & $(26,1)$ & $(12,1)$ \\
\hline & & & 1 & $(38,1)$ & $(35,1)$ & $(18,1)$ & $(10,1.5)$ & $(37,2)$ & $(43,2)$ \\
\hline & & & & $(38,3)$ & $(33,3.5)$ & $(28,1.5)$ & $(22,2)$ & $(38,5)$ & $(12,3)$ \\
\hline & & & 2 & $(38,2)$ & $(43,2)$ & $(28,2)$ & $(19,2.5)$ & $(43,10)$ & $(43,4)$ \\
\hline & & \multirow{4}{*}{2} & & $(38,2)$ & $(35,4)$ & $(22,1.5)$ & $(22,2)$ & $(12,2)$ & $(12,1)$ \\
\hline & & & 1 & $(38,3)$ & $(35,2)$ & $(38,3)$ & $(38,3.5)$ & $(17,3)$ & $(73,2)$ \\
\hline & & & & $(38,2.5)$ & $(38,3)$ & $(23,1)$ & $(23,1.5)$ & $(21,4)$ & $(31,3)$ \\
\hline & & & 2 & $(38,3.5)$ & $(38,2)$ & $(38,2)$ & $(18,2.5)$ & $(43,5)$ & $(73,4)$ \\
\hline
\end{tabular}


Table 6. Minimum and maximum values of the numerator and denominator of all objectives

\begin{tabular}{|c|c|c|c|c|}
\hline \multirow{2}{*}{ Levels } & \multicolumn{2}{|c|}{ Objectives } & Max & Min \\
\hline \multirow{2}{*}{1} & \multirow{2}{*}{$Z^{(1)}$} & Numerator & $\bar{N}^{(1)}=46570$ & $\underline{N}^{(1)}=23625$ \\
\cline { 3 - 5 } & & Denominator & $\bar{D}^{(1)}=50960$ & $\underline{D}^{(1)}=21285$ \\
\hline \multirow{2}{*}{2} & \multirow{2}{*}{$Z^{(2)}$} & Numerator & $\bar{N}^{(2)}=29760$ & $\underline{N}^{(2)}=13765$ \\
\cline { 3 - 5 } & & Denominator & $\bar{D}^{(2)}=41315$ & $\underline{D}^{(2)}=11710$ \\
\hline \multirow{2}{*}{3} & \multirow{2}{*}{$Z^{(3)}$} & Numerator & $\bar{N}^{(3)}=44555$ & $\underline{N}^{(3)}=7340$ \\
\cline { 3 - 5 } & & Denominator & $\bar{D}^{(3)}=62035$ & $\underline{D}^{(3)}=15565$ \\
\hline
\end{tabular}

$\delta=0, x_{11111}=140, x_{12221}=60, x_{21121}=$

$10, x_{21122}=125, x_{22111}=140, x_{22222}=$ $300, d_{N^{+}}^{1}=d_{N^{+}}^{2}=d_{N^{+}}^{3}=d_{D^{+}}^{1}=d_{D^{+}}^{2}=d_{D^{+}}^{3}=$ $0, d_{N^{-}}^{1}=0.20, d_{N^{-}}^{2}=0.27, d_{N^{-}}^{3}=0.32, d_{D^{-}}^{1}=$ $0.69, d_{D^{-}}^{2}=0.90, d_{D^{-}}^{3}=0.57$

with the corresponding objective values $Z^{(1)}=$ $0.933, Z^{(2)}=1.246, Z^{(3)}=0.548$.

Repeat the above said steps from 1 to 5 for chance constraint method for the proposed UML4DMIFTP to obtain the compromise solution of the model. The solution has been obtained by considering predetermined confidence level as: $\alpha_{n}=\gamma_{n}=$ $\alpha_{s w}=\gamma_{d w}=\beta_{v}=0.9 \forall n, v, s, w \& d$.

The compromise solution has been given as follows $Z^{(1)}=0.86, Z^{(2)}=1.146, Z^{(3)}=0.648$. The decision makers can obtain the optimal solutions flexibly, as per their desired conditions, by using the chance constrained method. Considering diverse set of values for various parameters in the proposed model, will benefit the decision making under uncertain environment.

\section{Conclusion}

A network planning model has been presented for multi level four diemension muti item fractional transportation problem under uncertain variables on this paper. Multi level four diemensional multi item fractional transportation problem to handle real life situations has been discussed for the first time ever in this paper. The transportation problem discussed in the work has been considered by using fractional programming rather than the linear programming, we consider ratio of two linear functions to optimize which also yields us the efficiency of the system. The problem also considers decision making at various level i.e. hierarchical decision making system. It also eliminates the deadlock situations where contradicting objectives exist using goal programming method. The numerical example has been taken and solved to illustrate the solution procedure and also the validity of the model presented. The proposed model, unlike the others till date, can generally be used for any type of 2D/3D/4D fractional transportation problem and also yields the efficiency of the system. The above work can be extended by including multiple objectives in each level of decision making in the future.

\section{Conflict of interests}

The authors declare that there is no conflict of interests regarding the publication of this paper.

\section{Author contributions}

Conceptualization, A. N. Revathi and S. Mohanaselvi; methodology, A. N. Revathi and S. Mohanaselvi; software, A. N. Revathi; validation, S. Mohanaselvi and A. N. Revathi; formal analysis, S. Mohanaselvi; investigation, A. N. Revathi and S. Mohanaselvi; resources, A. N. Revathi and S. Mohanaselvi; data-curation, A. N. Revathi and S. Mohanaselvi; writing - original draft preparation, A. N. Revathi; writing - review and editing, A. N. Revathi ; visualization, A. N. Revathi and S. Mohanaselvi; supervision, S. Mohanaselvi”.

\section{References}

[1] F. L. Hitchcock, "The distribution of a product from several sources to numerous localities", Journal of Mathematical Physics, Vol. 20, pp. 224-330, 1941.

[2] L. Kaur, Madhuchanda, and S. Sandeep, "A new approach to solve multi objective transportation problem", Applications and applied mathematics, Vol. 13, No. 1, pp. 150159, 2018.

[3] K. B. Haley, "New methods in mathematical programming- The solid transportation 
problem”, Operation Research, Vol. 10, No. 4, pp. 448-463, 1962.

[4] H. L. Bhatia, "Indefinite quadratic solid transportation problem", Journal of Information and Optimization Science, Vol. 2, No. 3, pp. 297-303, 1981.

[5] A. Charnes and W. W. Cooper, "Management models and industrial applications of linear programming", Management Science, Vol. 4, No. 1, pp. 38-91, 1957.

[6] K. Swarup, "Transportation technique in linear fractional functional programming", Journal of Royal Naval Scientific Service, Vol. 21, No. 5, pp. 256-260, 1966.

[7] A. Khurana and S. R. Arora, "The sum of a linear and a linear fractional transportation problem with restricted and enhanced flow", Journal of Interdisciplinary Mathematic, Vol. 9, No. 2, pp. 373-383, 2006.

[8] V. D. Joshi and N. Gupta, "Linear fractional transportation problem with varying demand and supply", Le Matematiche, Vol. 66, No. 2, pp. 3-12, 2011.

[9] S. Pramanik and P. P. Dey, "Bi-level linear fractional programming problem based on fuzzy goal programming approach", International Journal of Computer Applications, Vol. 25, No. 11, pp. 34-40, 2011.

[10] A. Sameeullah, S. D. Devi, and B. Palaniappan, "Genetic algorithm based method to solve linear fractional programming problem", Asian Journal of Information Technology, Vol. 7, No. 2, pp. 83-86, 2008.

[11] N. Guzel, "A proposal to the solution of multiobjective linear fractional programming problem", Hidnawi Publishing Corporation Abstract and Applied Analysis, vol. 2013, pp. 14, 2013.

[12] W. Dinkelbach, "On Nonlinear Fractional Programming", Management Science, Vol. 13, No. 7, pp. 492-498, 1967.

[13] V. E. Sobana and D. Anurohda, "Solution of solid transportation problem in fuzzy environment", International Journal of Pure Applied Mathematics, Vol. 119, No. 9, pp. 313321, 2018.

[14] D. P. Acharya, S. M. Kamaruzzaman, and A. Das, "Solving multi-objective generalized solid transportation problem by interactive fuzzy goal programming approach", International Journal of Scientific Research and Publication, Vol. 7, No. 7, pp. 778-786, 2017.

[15] D. Rani and T. R. Gulati, "Uncertain multiobjective multi-product solid transportation problems", Sadhana, Vol. 41, No. 5, pp. 531539, 2016.

[16] Dheyab, "Finding the optimal solution for fractional linear programming problems with fuzzy numbers", Journal of Kerbala University, Vol. 10, No. 3, pp. 105- 110, 2012.

[17] S. Jain, "Modeling of Gauss elimination technique for multi-objective fractional programming problem", South Asian Journal of Mathematics, Vol. 4, No. 3, pp. 148- 153, 2014.

[18] V. D. Joshi, "On a Paradox in Multi Objective Linear and Fractional Transportation Problem", Science \& Technology Asia, Vol. 25, pp. 157$165,2020$.

[19] D. Moumita and P. K. De , "A note on fuzzy multi-objective linear fractional programming problem", International Journal of Computer Science and Network, Vol. 3, No. 6, pp. 568-572, 2014.

[20] B. Liu, "Uncertainty Theory", 2nd ed., Springer-Verlag, Berlin, pp. 205-233, 2007.

[21] H. Guo, X. Wang, and S. Zhou, "A Transportation Problem with Uncertain Costs and Random Supplies", International Journal of e-Navigation and Maritime Economy, Vol. 2, pp. $1-11,2015$.

[22] S. M. Ghasemi and M. Safi, "Linear Fractional Programming Problem in Uncertain Environments", Journal of Mathematical Analysis. Vol. 8, No. 2, pp. 23-33, 2017.

[23] A. Mahmoodirad, R. Dehghan and S. Niroomand, "Modelling linear fractional transportation problem in belief degree-based uncertain environment", Journal of experimental \& theoretical artificial intelligence, Vol. 31, No. 3, pp. 393-408, 2019.

[24] A. N. Revathi and S. Mohanaselvi, "A new solution approach to the uncertain multiobjective linear fractional programming problems", In: Proc. of AIP Conference Proceedings 2277, pp. 200009-1-200009-8, 2020.

[25] B. Liu and K. Yao, "Uncertain multilevel programming: algorithm and applications", Computers \& Industrial Engineering, Vol. 89, pp. 235-240, 2015.

[26] Y. Gao and S. Kar, "Uncertain solid transportation problem with product blending", International Journal of Fuzzy Systems, Vol. 19, pp. 1916-1926, 2017.

[27] B. Zhang, J. Peng, S. Li, and L. Chen, "Fixed charge solid transportation problem in uncertain environment and its algorithm", Computers \& Industrial Engineering, Vol. 102, No. C, pp. 186-197, 2016. 
[28] H. Dalman, "Uncertain programming model for multi-item solid transportation problem", International Journal of Machine Learning and Cybernetics, Vol. 9, pp. 559-567, 2016.

[29] L. Cheng, C. Rao, and L. Chen, "Multidimensional knapsack problem based on uncertain measure", Scientia Iranica E, Vol. 24, No. 5, pp. 2527-2539, 2017.

[30] A. N. Revathi and S. Mohanaselvi, "Uncertain Multi-Objective Multi-Item Four Dimensional Fractional Transportation Model", Annals of the Romanian Society for Cell Biology, Vol. 25, No. 4, pp. 231-247, 2021.

[31] S. A. Jalil, S. Javaid, and S. M. Muneeb, "A decentralized multi-level decision making model for solid transportation problem with uncertainty", International Journal of System Assurance Engineering and Management, Vol. 9, No. 5, pp. 1022-1033, 2018.

[32] V. D. Josh1 and N. Gupta, "Linear fractional transportation problem with varying demand and supply", Le Matematiche, Vol. LXVI, Fasc. II, pp. 3-12, 2011.

[33] K. Lachhwani and M. P. Poonia, "Mathematical solution of multilevel fractional programming problem with fuzzy goal programming approach", Journal of Industrial Engineering International, Vol. 8, pp. 1-11, 2012.

[34] S. T. Liu, "Fractional transportation problem with fuzzy parameters", Soft Computing, Vol. 20, No. 9. pp. 3629-3636, 2015.

[35] S. Sadia, Q. Ali, and N. Gupta, "Multi objective fractional transportation problem in fuzzy environment", International Journal of Mathematical Archive, Vol. 8, No. 12, pp. 197209, 2017.

[36] S. A. Jalil, S. Javiad, and Z. A. Sim, "A model for uncertain multi objective transportation problem with fractional objectives", International Journal of Operation Research, Vol. 14, No. 1, pp. 11-25, 2017.

[37] M. S. Osman, O. E. Emam, and M. L. Elsayed, "Interactive Approach for Multi-Level MultiObjective Fractional Programming Problems with Fuzzy Parameters", Beni-Suef University Journal of Basic and Applied Sciences, Vol. 7, No. 1, pp. 139-149, 2018.

[38] J. M. Vinotha, W. Ritha, and I. A. Vinoline, “An algorithm for multi objective fuzzy fractional transportation problem", European Journal of Molecular \& Clinical Medicine, Vol. 07, No. 09, pp. 3142-3153, 2020.

[39] H. A. E. W. Khalifa, P. Kumar, and M. G. Alharbi, "On characterizing solution for multi- objective fractional two-stage solid transportation problem under fuzzy environment", Journal of Intelligent Systems, Vol. 30, pp. 620-635, 2021.

[40] K. Lachhwani and S. Nehra, "Modified FGP approach and MATLAB program for solving multi-level linear fractional programming problems", Journal of Industrial Engineering International, Vol. 11, pp. 15-36, 2015.

[41] B. Liu, "Some Research Problems in Uncertainty Theory", Journal of Uncertain Systems, Vol. 3, No. 1, pp. 3-10, 2009.

[42] B. Liu, "Uncertainty Theory, A Branch of Mathematics for Modeling Human Uncertainty", Springer-Verlag, Berlin, 2010.

[43] Y. Liu and M. Ha, "Expected values of function of uncertain variables", Journal of Uncertain Systems, Vol. 4, No. 3, pp. 181-186, 2010.

[44] A. Charnes and W. W. Cooper, "Goal Programming and Multiple objective", European Journal of Operation Research, Vol. 1, No. 1, pp. 39-54, 1977.

[45] T. Chang, "Muti-choice goal programming," Omega, Vol. 35, No. 4, pp. 389-396, 2007.

[46] B. B. Pal, B. N. Moitra, and U. Maulik, "A goal programming procedure for fuzzy multiobjective linear fractional programming problem”, Fuzzy Set Systems, Vol. 139, pp. 395405, 2003.

[47] R. H. Mohamed, "The relationship between goal programming and fuzzy programming", Fuzzy Sets and Systems, Vol. 89, Issue.2, pp. 215-222, 1997.

[48] M. Zangiabadi and H. R. Maleki, "Fuzzy goal programming for multi objective transportation problem," Journal of Applied mathematics and Computing, Vol. 24, No. 1-2, pp. 449-460, 2007.

[49] M. Zangiabadi and H. R. Maleki, "Fuzzy goal programming technique to solve multi objective transportation problem with some non-linear membership function", Iranian Journal of Fuzzy Systems, Vol. 10, pp. 61-74, 2013. 